\title{
Bilayer Quantum Hall Systems at Filling Factor $\nu=2$ : An Exact Diagonalization Study
}

\author{
John Schliemann ${ }^{1, *}$ and A.H. MacDonald ${ }^{2}$ \\ ${ }^{1}$ Physikalisches Institut, Universität Bayreuth, D-95440 Bayreuth, Germany \\ ${ }^{2}$ Department of Physics, Indiana University, Bloomington, Indiana 47405
}

(Received 17 December 1999)

\begin{abstract}
We present an exact diagonalization study of bilayer quantum Hall systems at filling factor $\nu=2$ in the spherical geometry. We find the high-Zeeman-coupling phase boundary of the broken symmetry canted antiferromagnet is given exactly by previous Hartree-Fock mean-field theories, but that the state's stability at weak Zeeman coupling has been qualitatively overestimated. In the absence of interlayer tunneling, degeneracies occur between total spin multiplets due to the Hamiltonian's invariance under independent spin rotations in top and bottom two-dimensional electron layers.
\end{abstract}

PACS numbers: $73.40 . \mathrm{Hm}$

In the last decade there has been an increasing interest in quantum Hall ferromagnets [1]. Most recently bilayer systems at a filling factor of 2 have become the object of intensive theoretical [2-8] and experimental [9-11] research. The rich phenomenology of $\nu=2$ bilayers mirrors a complex interplay between Coulomb interactions in the lowest Landau level, Fermi statistics, and the coupling of external fields to spin and layer degrees of freedom.

Our current microscopic understanding of $\nu=2$ bilayer quantum Hall ferromagnets is based on Hartree-Fock mean-field theory calculations [2,3], and on a partially phenomenological effective spin Hamiltonian description [4]. Both approaches lead to the prediction of a novel broken symmetry canted-antiferromagnet ground state with finite spin susceptibility which interpolates, as external field parameters are varied, between a fully spin-polarized state and a spin-singlet state, both of which have charge and spin gaps and zero differential spin susceptibility. In this Letter we report on the first exact diagonalization study of finite bilayer systems at filling factor $\nu=2$. Our calculations support the predicted occurrence of a broken symmetry ground state, and indeed demonstrate that the Hartree-Fock result for its large Zeeman-coupling phase boundary is exact. We find that the stability of the cantedantiferromagnet state relative to the spin-singlet state is overstated by the Hartree-Fock approximation, and estimate the correct position of the phase boundary.

A bilayer system in a strong magnetic field is described in spherical geometry by the following Hamiltonian:

$$
\mathcal{H}=\mathcal{H}_{1 \mathrm{P}}+\mathcal{H}_{\text {Coul }} \text {, }
$$

where $\mathcal{H}_{\text {Coul }}$ represents the usual Coulomb interaction within and between layers, and the single-particle Hamiltonian $\mathcal{H}_{1 \mathrm{P}}$ is given by

$$
\begin{aligned}
\mathcal{H}_{1 \mathrm{P}}= & -\frac{1}{2} \sum_{m} c_{\mu, \sigma, m}^{+}\left[\Delta_{v} \tau_{\mu, \mu^{\prime}}^{z} \delta_{\sigma, \sigma^{\prime}}\right. \\
& \left.\quad+\Delta_{t} \tau_{\mu, \mu^{\prime}}^{x} \delta_{\sigma, \sigma^{\prime}}+\Delta_{z} \delta_{\mu, \mu^{\prime}} \sigma_{\sigma, \sigma^{\prime}}^{z}\right] \\
& \times c_{\mu^{\prime}, \sigma^{\prime}, m} .
\end{aligned}
$$

A summation convention is understood for repeated Greek indices, where $\mu, \mu^{\prime} \in\{+,-\}$ run over the layer (or pseudospin) index, while $\sigma, \sigma^{\prime} \in\{\uparrow, \downarrow\}$ run over the $z$ projections of the electron spin; $\vec{\tau}$ and $\vec{\sigma}$ are Pauli matrices for pseudospin and electron spin, respectively. $m \in\left\{-N_{\phi} / 2, \ldots, N_{\phi} / 2\right\}$ is the $z$ projection of the orbital angular momentum of each electron in the lowest Landau level, where $N_{\phi}$ is the number of flux quanta penetrating the sphere. The Hamiltonian contains bias voltage $\left(\Delta_{v}\right)$, tunneling $\left(\Delta_{t}\right)$, and Zeeman-coupling $\left(\Delta_{z}\right)$ terms. In the following we measure the interlayer separation $d$ in units of the magnetic length $l_{B}=\sqrt{\hbar c / e B}$ and all energies in units of the Coulomb energy scale $e^{2} / \epsilon l_{B}$.

We first consider the case where all single-particle coupling constants vanish. The number of particles in each layer is then a good quantum number and, in the ground state, both layers have a filling factor of 1 . Moreover, because the Coulomb interaction is spin independent, the Hamiltonian is invariant under independent spin rotations in either layer. It follows that the total spin in either layer is a good quantum number.

For layer separation $d \rightarrow \infty$ it is known that the ground state of each isolated layer is a quantum Hall ferromagnet with $S=N / 4$, where $N$ is the total number of particles in the two layers. Since the Coulomb interaction within the layers is stronger than between them, we anticipate that this should remain true at any finite $d$. Indeed, this expectation is confirmed numerically. The upper diagram of Fig. 1 shows the low-lying spectrum of a double layer system at a filling factor of 2 with eight electrons in the case of vanishing single-particle couplings. The ground state as described there consists of multiplets with total spin quantum number $S$ varying from 0 to 4 , the total spin representations of a state with good spin quantum number $S=2$ in each layer. When an interlayer tunneling term is added to the Hamiltonian, the additional symmetry responsible for these degeneracies is lost and only total spin is a good quantum number.

The robustness of individual layer ground state spin quantum numbers against the effects of added interlayer interactions is not restricted to a total filling factor of 2 . In fact, in our finite-size numerical calculations, it holds at all combinations of numbers of flux quanta and electrons we have checked. 


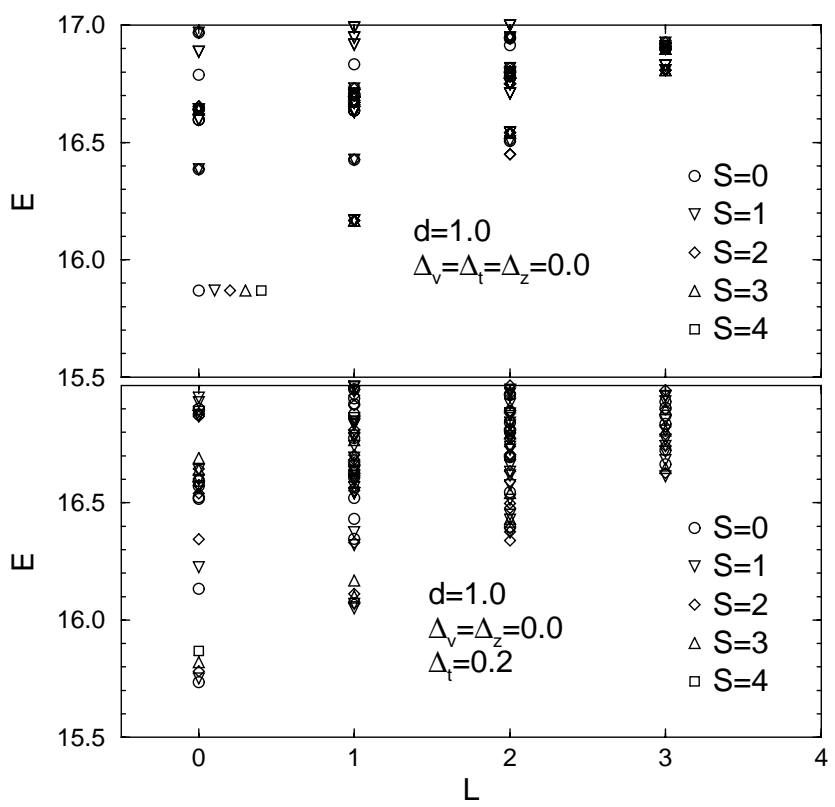

FIG. 1. The low-lying spectrum of a double layer system at a filling factor of 2 with eight electrons. The energy levels are plotted as a function of the total orbital angular momentum $L$ for different values of the total spin $S$. The upper diagram shows the case of vanishing single-particle couplings, while in the lower spectrum $\Delta_{t}$ is finite. For $\Delta_{t}=0$, the degenerate spin multiplets in the ground state are exhibited explicitly.

For instance, consider the system discussed above, but with one electron either added or removed. (These two cases are equivalent by particle-hole symmetry.) In the ground state, one of the layers has a filling factor of 1 (a ground state with $L=0, S=2$ ), while the other one contains a hole and the low-lying states are organized in the well-known Skyrmion branch having quantum numbers $L=S=1 / 2,3 / 2$ [12]. The spectrum of this double layer system is shown in the upper diagram of Fig. 2. The ground state carries quantum numbers $L=1 / 2$ and $S=3 / 2, S=5 / 2$ and is the result of coupling the $L=0, S=2$ ground state of the full layer to the $L=S=1 / 2$ ground state of the hole system. Since either layer can carry the hole, we get two copies of the degenerate multiplets. The next higher degenerate group of multiplets is the result of coupling the ground state of the $\nu=1$ layer to the $L=S=3 / 2$ state of the hole layer, and again all multiplets are doubled. To confirm this interpretation we have verified that this degeneracy doubling is lifted by applying a bias voltage, as shown in the lower diagram of Fig. 2. These results demonstrate that in the absence of interlayer tunneling, bilayer states for $\nu$ near 2 can be safely regarded as two single-layer quantum Hall ferromagnets whose coupling has only a quantitative significance, for example, in changing the energies of the Skyrmionic elementary charged excitations.

With this established, we now focus on changes in the nature of the ground state at $\nu=2$ as the single-particle coupling constants are varied. An arbitrarily small tunnel- ing amplitude is enough to break the degeneracy among the different spin multiplets and make the spin-singlet state with the most antiparallel electron spin structure and consequently the most parallel pseudospin structure the nondegenerate ground state. More precisely, the energy levels are found to be ordered by the total spin $S$ with the difference between neighboring levels increasing with increasing $S$, as shown in the lower diagram of Fig. 1. We note that the lone maximally polarized $S=N / 2$ multiplet is annihilated by the interlayer tunneling term in the Hamiltonian and has an eigenenergy which is independent of $\Delta_{t}$. Turning on the Zeeman coupling $\Delta_{z}$ at a given value of the tunneling $\Delta_{t}$ does not change the eigenstates themselves, but only shifts their energies and breaks the degeneracy within each spin multiplet.

These findings lead to the following scenario: With increasing $\Delta_{z}$ a lower critical Zeeman coupling $\Delta_{z}^{(1)}\left(\Delta_{t}\right)$ is reached where the state with $S^{z}=S=1$ becomes the ground state, i.e., the system leaves the spin-singlet phase. If $\Delta_{z}$ is increased further, the ground state $S^{z}=S$ quantum number increases monotonously until, at an upper critical value $\Delta_{z}^{(2)}\left(\Delta_{t}\right)$, the fully spin-polarized state $S^{z}=$ $S=N / 2$ is reached.

Finite-size spectra are shown in Fig. 3 for several Zeeman couplings at $\Delta_{t}=0.8$. In the top diagram the system is in the spin-singlet phase, while in the bottom the ground state is fully spin polarized. In the narrow transition area $\Delta_{z}^{(1)} \leq \Delta_{z} \leq \Delta_{z}^{(2)}$ all low-lying states with $S^{z}=0, \ldots, N / 2$ have energies very close to each other. This holds also for a branch of states with angular momentum $L>0$ which appears to be separated by a gap from higher-lying parts of the spectrum. We identify this transition region with the canted-antiferromagnetic

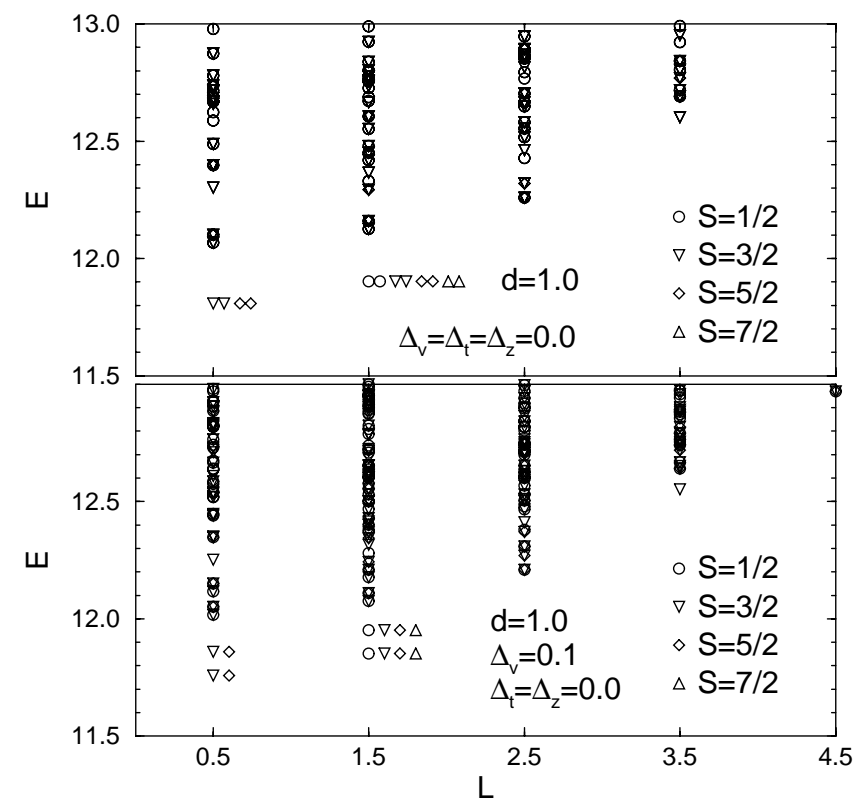

FIG. 2. The same system as in Fig. 1, but with one electron removed. The degenerate multiplets discussed in the text are shown in detail. 

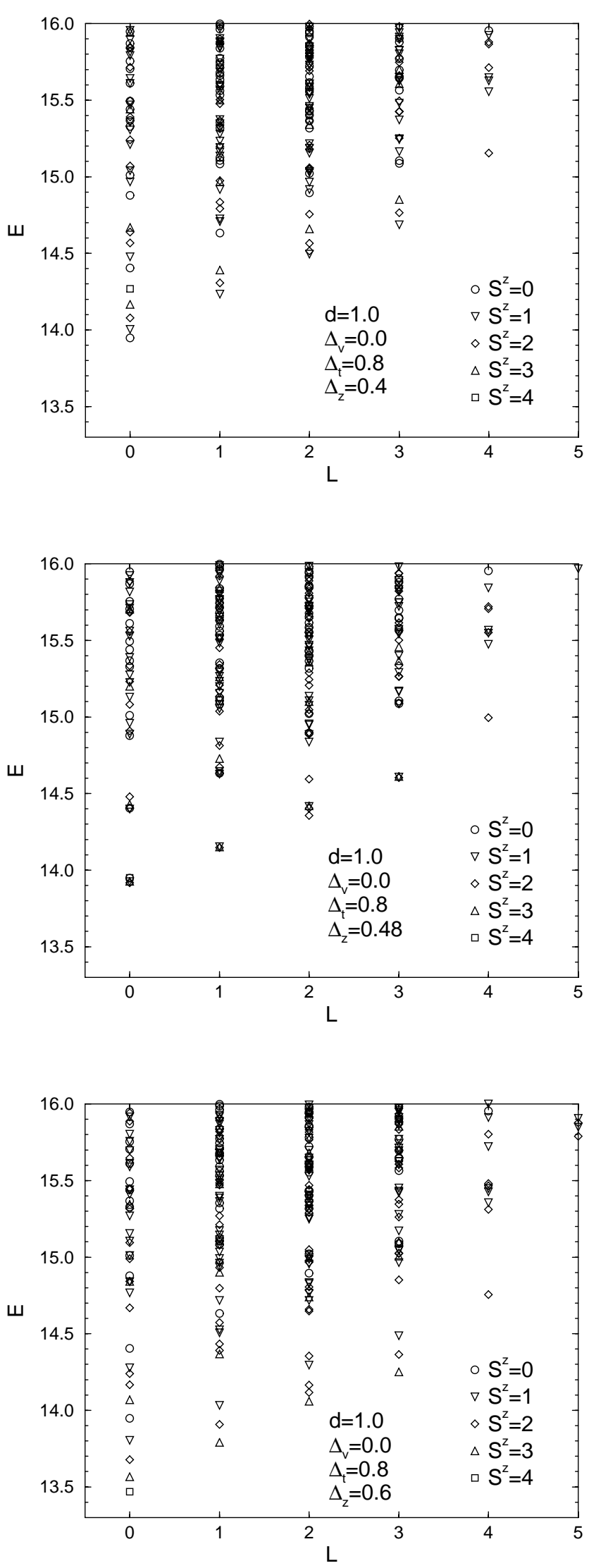

FIG. 3. Finite-size spectra for a system of eight electrons as a function of the Zeeman coupling for tunneling amplitude $\Delta_{t}=$ 0.8 . States with negative values of $S^{z}$ are omitted for clarity. See text for details. phase first proposed by Zheng et al. on the basis of the unrestricted Hartree-Fock approximation [2].

The mean-field state, which breaks spin-rotational symmetry around the $\hat{z}$ axis can be constructed as a linear combination of the nearly degenerate exact eigenstates $\left|S^{z}\right\rangle$ carrying quantum numbers $L=0$ and definite $S^{z}$ values. To analyze the spin structure perpendicular to the Zeeman axis we introduce spin operators for each layer separately, $\vec{S}_{\mu}, \mu \in\{+,-\}$, where the total spin of the bilayer system is given by $\vec{S}=\sum_{\mu} \vec{S}_{\mu}$. Since the states $\left|S^{z}\right\rangle$ belong to different multiplets, all matrix elements of $S^{ \pm}$between them are zero, which means that

$$
\left\langle S^{z}\left|S_{\mu}^{+}\right| S^{z}-1\right\rangle=-\left\langle S^{z}\left|S_{-\mu}^{+}\right| S^{z}-1\right\rangle .
$$

Thus, the matrix elements of spin components perpendicular to the Zeeman axis have the same magnitude and opposite sign in opposite layers. Any wave packet constructed from these states will, like the mean-field state, have opposite transverse spin polarization in the two layers.

Next let us consider the phase diagram, i.e., the functions $\Delta_{z}^{(1)}\left(\Delta_{t}\right)$ and $\Delta_{z}^{(2)}\left(\Delta_{t}\right)$. In the thermodynamic limit these lines mark the boundaries between spin-polarized, spin-singlet, and canted-antiferromagnet phases. They may be compared with the Hartree-Fock results obtained recently in the planar geometry for an infinite system [3]. To avoid unnecessary finite-size uncertainty, we have rederived the Hartree-Fock equations for the spherical geometry obtaining explicit expressions for finite systems. We find that the phase boundaries have the same form as for the infinite system [3]:

$$
\Delta_{z}^{(1)}=\sqrt{\Delta_{t}\left(\Delta_{t}-2 F_{-}\right)}
$$

for $\Delta_{t}>2 F_{-}$, otherwise $\Delta_{z}^{(1)}=0$, and

$$
\Delta_{z}^{(2)}=\sqrt{\Delta_{t}^{2}+F_{-}^{2}}-F_{-} .
$$

In the present case, however, the exchange parameter $F_{-}$ is size dependent:

$$
F_{-}=\frac{e^{2}}{\epsilon l_{B}} \frac{N_{\phi}+1}{\sqrt{2 N_{\phi}}}\left[I(1)-\left(\frac{1}{\alpha}\right)^{N_{\phi}+1 / 2} I(\alpha)\right],
$$

with

$$
I(\alpha)=\int_{0}^{\alpha} d x \frac{x^{N_{\phi}}}{\sqrt{1-x}}, \quad \alpha=\frac{1}{1+\frac{1}{N_{\phi}} \frac{d^{2}}{2 l_{B}^{2}}} .
$$

In Fig. 4 we compare exact diagonalization and HartreeFock finite-size phase boundaries for $N=6,8,10$, and 12 electrons. Not unexpectedly, the region of the cantedantiferromagnetic phase turns out to be much smaller than predicted by the Hartree-Fock theory. Interestingly however, the two results for the phase boundary $\Delta_{z}^{(2)}\left(\Delta_{t}\right)$ coincide within our numerical precision of at least $10^{-12}$. Since both the spin-polarized state and its elementary collective excitations, which go soft at the phase boundary, are described exactly [13] by Hartree-Fock theory, this coincidence is not entirely unexpected. It does, however, very convincingly demonstrate that the spin-polarized to canted phase transition remains continuous when quantum 


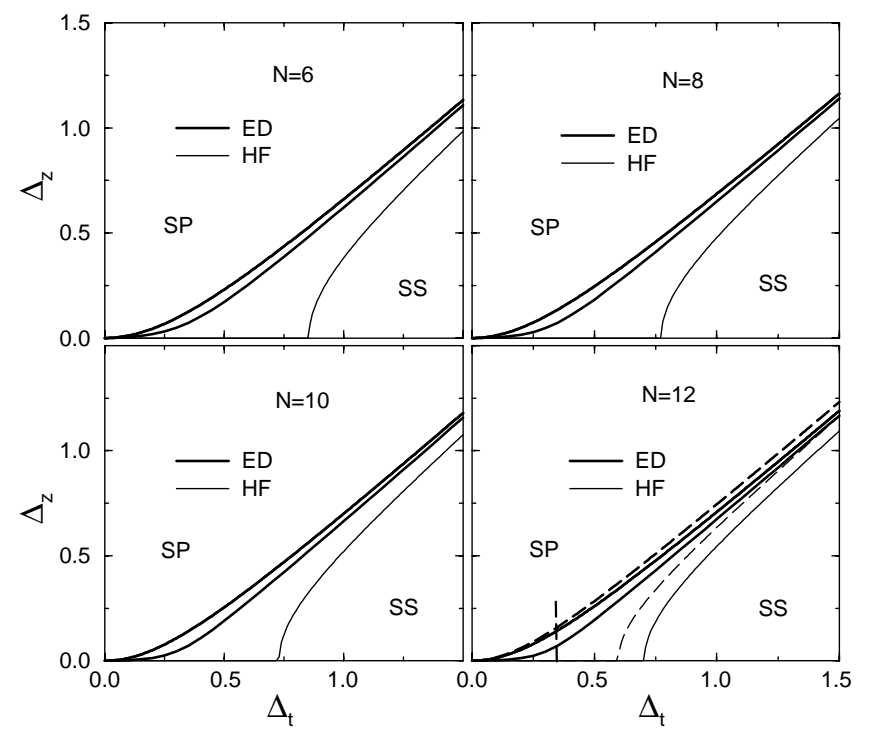

FIG. 4. Phase diagrams at a layer separation of $d=1.0$ and zero bias voltage for different system sizes. The upper and lower critical Zeeman couplings as obtained from exact diagonalization (ED) data are plotted as thick lines. The cantedantiferromagnetic phase lies between them and separates the spin-polarized phase (SP) from the spin-singlet phase (SS). The finite-size Hartree-Fock (HF) results are given for comparison. The upper HF phase boundary coincides with the ED result (see text), while the lower phase boundary strongly overestimates the region of the canted-antiferromagnetic ground state. The last panel $(N=12)$ shows in addition the HF phase boundaries for the infinite system (where the upper one is as well exact) as dashed lines. The value of $\Delta_{t}$ below which the ED results for $\Delta_{z}^{(1)}\left(\Delta_{t}\right)$ decrease with system size is marked by a vertical line.

fluctuations are included. We conclude that the position of this phase boundary in the thermodynamic limit can be calculated exactly using the expressions given in Ref. [3], or equivalently the $N_{\phi} \rightarrow \infty$ limit of Eqs. (5)-(7), adding finite well width and other sample-specific corrections as required.

The result for this upper boundary is in marked contrast with our findings for the lower boundary. Here the Hartree-Fock approach leads to a canted antiferromagnetic region at all system sizes even in the absence of the Zeeman coupling. A similar conclusion was reached by Demler and Das Sarma using the effective spin theory [4]. In our finite-size exact diagonalization calculations, on the other hand, $\Delta_{z}^{(1)}$ is always finite. For $\Delta_{t} \lesssim 0.35$, marked by a vertical line in the last panel of Fig. $4, \Delta_{z}^{(1)}$ decreases monotonously with system size, while it increases monotonously for larger values of $\Delta_{t}$. These observations guarantee that the canted-antiferromagnetic phase predicted by Hartree-Fock theory is actually present in the infinite system (although clearly diminished by quantum fluctuations). Moreover, our findings are consistent with a vanishing thermodynamic limit of $\Delta_{z}^{(1)}\left(\Delta_{t}\right)$ and a nonzero spin susceptibility for $\Delta_{t} \lesssim 0.35$. Static correlation function calculations [14] at $\Delta_{z}=0$ are also consistent with a nonzero canted order parameter for $\Delta_{t}<0.35$. These numerical results are thus consistent with a single inter- mediate phase which has both a finite spin susceptibility and canted-antiferromagnet order. For $\Delta_{t} \geqslant 0.35, \Delta_{z}^{(1)}$ increases slowly with system size, presumably saturating at a finite value smaller than $\Delta_{z}^{(2)}$ and leaving a narrow canted-antiferromagnet strip in the phase diagram. The maximum $\Delta_{t}$ value at which the ordered state phase extends down to $\Delta_{z}=0$ is much smaller than the value $\Delta_{t}=0.60$, predicted by Hartree-Fock theory. These findings are illustrated along with the exact upper phase boundary in the right bottom panel of Fig. 4, which shows that the Hartree-Fock approximation strongly overestimates the stability of the canted-antiferromagnetic phase against the spin-singlet phase in finite systems and as well in the thermodynamic limit.

J.S. acknowledges support from the Deutsche Forschungsgemeinschaft under Grant No. SCHL 539/1-1. A. H. M. was supported by the National Science Foundation under Grant No. DMR-9714055.

*Email address: btp338@theo.phy.uni-bayreuth.de Present address: Department of Physics, Indiana University, Bloomington, IN 47405.

[1] For a review on experimental research on multicomponent quantum Hall systems, see J. P. Eisenstein, in Perspectives in Quantum Hall Effects, edited by S. Das Sarma and A. Pinczuk (Wiley, New York, 1997); for a review on theoretical research, see S.M. Girvin and A.H. MacDonald in the same volume. For a general discussion of quantum Hall ferromagnets, see also T. Jungwirth and A. H. MacDonald (to be published).

[2] L. Zheng, R. J. Radtke, and S. Das Sarma, Phys. Rev. Lett. 78, 2453 (1997); S. Das Sarma, S. Sachdev, and L. Zheng, Phys. Rev. Lett. 79, 917 (1997); Phys. Rev. B 58, 4672 (1998).

[3] A. H. MacDonald, R. Rajaraman, and T. Jungwirth, Phys. Rev. B 60, 8817 (1999).

[4] E. Demler and S. Das Sarma, Phys. Rev. Lett. 82, 3895 (1999).

[5] L. Brey, E. Demler, and S. Das Sarma, Phys. Rev. Lett. 83, 168 (1999).

[6] E. Demler, E.H. Kim, and S. Das Sarma, cond-mat/ 9907107.

[7] K. Yang, Phys. Rev. B 60, 15578 (1999).

[8] M.-F. Yang and M.-C. Chang, Phys. Rev. B 60, 13985 (1999); cond-mat/9909282.

[9] V. Pellegrini, A. Pinczuk, B. S. Dennis, A. S. Plaut, L. N. Pfeiffer, and K. W. West, Phys. Rev. Lett. 78, 310 (1997); Science 281, 799 (1998).

[10] A. Sawada, Z.F. Ezawa, H. Ohno, Y. Horikoshi, Y. Ohno, S. Kishimoto, F. Matsukara, M. Yasumoto, and A. Urayama, Phys. Rev. Lett. 80, 4534 (1998).

[11] V.S. Khrapai, E. V. Deviatov, A. A. Shashkin, V.T. Dolgopolov, F. Hastreiter, A. Wixforth, K. L. Campman, and A. C. Gossard, cond-mat/9903396.

[12] X. C. Xie and S. He, Phys. Rev. B 53, 1046 (1996).

[13] See, for example, A. H. MacDonald, J. Phys. C 18, 1003 (1985).

[14] John Schliemann and A. H. MacDonald (unpublished). 